\title{
Machines à sous
}

\section{Erhard Taverna}

Dr med., membre de la rédaction

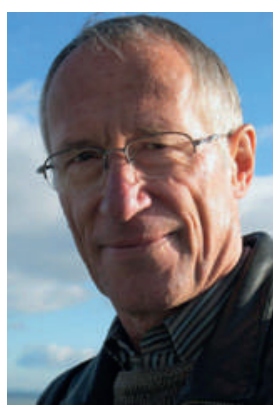

Les déficits des groupements d'hôpitaux entraînent réprimandes au parlement cantonal, plus ou moins sévères suivant le parti politique, propositions de fusion, surveillance accrue et recherche de prestations plus rentables. Les excédents, eux, respectent la consigne de recherche de bénéfices. En 2014, l'hôpital cantonal de Lucerne a enregistré 52 millions de bénéfices annuels [1], dont 14 seraient partis dans les caisses du canton, bien que l'organisation santésuisse se soit prononcée contre les financements croisés. Suivent, en haut de la liste, les Grisons avec 36 millions, l'hôpital universitaire de Zurich avec 28, l'Inselspital avec 25, Winterthour avec 24, Baden avec 23, l'hôpital universitaire de Bâle avec 14 et Saint-Gall avec 7.

Purs gains de productivité? C'est à se demander si les payeurs de primes ne verseraient pas des contributions n'ayant rien à voir avec les soins médicaux. Beaucoup reconnaissent les effets pervers sur les hôpitaux du système DRG introduit il y a quatre ans. Le directeur de Insel AG, un regroupement d'hôpitaux comptant plus de 10000 collaborateurs, prône les fusions d'hôpitaux avec mandats échelonnés, malgré les fortes controverses à ce sujet. Il identifie un manque de volonté de régulation, par exemple lors de la fermeture du Zieglerspital de Berne, canton dans lequel le même nombre de nouveaux lits est autorisé en même temps dans un hôpital privé [2].

Chaque acteur du système de santé pense d'abord à son propre bilan. Diverses causes sont invoquées pour se dédouaner: interventions étatiques, liste d'hôpitaux dans les cantons, mise sur le même plan des hôpitaux publics et privés, débordements administratifs, hiérarchies inutiles, pyramide des âges, industrie pharmaceutique, nouvelles technologies, etc. Tant que les médecins revendiqueront un rôle de leaders, ils seront accusés en premier de générer des coûts. Nul ne conteste que les patients sont trop souvent opérés, par exemple en fin de vie. Cela tient d'une part à des raisons financières (bonus, objectifs et pots de vin), d'autre part, pour les partisans de la preuve scientifique, au manque d'études scientifiques (comparaisons de méthodes ou analyses à long terme). Une opération valant déjà comme le plus efficace des placébos, il est difficile de faire de la recherche en chirurgie. Une autorégulation, sur la base de ce qu'on appelait les listes noires et dans le sens d'une Smarter Medicine, peut générer quelques succès, mais ne devrait pas influencer l'essentiel du budget. On sait depuis longtemps ce qu'il faudrait améliorer d'un point de vue médical. Une étude du New England Journal of Medicine sur les résultats d'IRM de genoux de près d'un millier de patients, avec ou sans douleurs, a démontré que les ruptures méniscales étaient très fréquentes chez les personnes en bonne santé (notamment une personne de plus de 70 ans sur deux, sans symptômes). Avec la tomographie à résonance magnétique nucléaire, il devient difficile de distinguer les personnes en bonne santé des malades [3]. Quand de très légères altérations anatomiques sont visibles, orthopédistes et radiologues mettent en garde contre les interprétations erronées et les décisions thérapeutiques hâtives. Quand l'IRM en tenseur de diffusion révèle le moindre choc superficiel du cerveau, la tentation est grande pour mettre en rapport tous les symptômes possibles. Sauf quand une intervention en urgence est indiquée, l'IRM des patients souffrant de douleurs dorsales aiguës avec suspi-

C'est à se demander si les payeurs de primes ne verseraient pas des contributions n'ayant rien à voir avec les soins médicaux.

cion de hernie discale n'a aucun avantage à long terme. D'autres études réalisées ces dix dernières années ont montré qu'au bout de six mois, pour deux patients sur trois, la hernie avait nettement diminué, voire disparu, sans aucune intervention.

On peut comprendre les images et décider de ne rien faire et d'attendre. Des études vont dans ce sens dans toutes les spécialités, toujours plus nombreuses et solides. Ces travaux étaient déjà de bonne qualité il y a quelques années, sans que leurs auteurs en recueillent les lauriers. Certes, chacun écoute le prêche, mais dehors les règles ne sont pas les mêmes. Les intérêts personnels, une pression sur les rendements, attisée par la politique, et les exigences exagérées des patients empêchent temporairement que ces critères objectifs soient économiquement efficaces.

\section{Références}

1 K-Tipp du 20.4.2016, Christian Koutecky, Prämienzahler müssen Spital-Gewinne finanzieren

2 ZEIT ONLINE, 10.03.2016, Holger Baumann, Das ist ein Skandal.

3 Frankfurter Allgemeine Sonntagszeitung, 22.05.2016, Wer ist schon gesund? 